\title{
Biphasic dissolution method for quality control and assurance of drugs containing active substances in the form of weak acid salts
}

\author{
ALEŠ FRANC ${ }^{1}$ \\ JAN MUSELÍK ${ }^{1 *}$ \\ ROMAN GONĚC ${ }^{1,2}$ \\ DAVID VETCHÝ 1 \\ ${ }^{1}$ Department of Pharmaceutics \\ Faculty of Pharmacy \\ University of Veterinary \\ and Pharmaceutical Sciences \\ Brno, Czech Republic \\ ${ }^{2}$ Masaryk Memorial Cancer Institute \\ Brno, Czech Republic
}

Accepted September 29, 2015

Online published December 3, 2015

\begin{abstract}
Substances in the form of weak acid salts have been found to be problematic for dissolution testing. Their absorption can start only after they are turned into the form of an acid following the gastric passage although they were administered in the form of a salt. Due to poor solubility, they cannot be tested in acidic gastric environment for a biased dissolution profile. The biphasic dissolution method is promising for overcoming this obstacle. Tablets with warfarin clathrate sodium salt in two concentrations and two different particle size distributions were tested as a suitable model for finding the medium and process conditions of dissolution. The dissolution method based on the use of the upper organic layer (1-octanol) and the lower aqueous layer $\left(0.1 \mathrm{~mol} \mathrm{~L}^{-1} \mathrm{HCl}\right)$ was found suitable and discriminatory for tablets containing active substances in the form of salts of weak acids. The method also reflects physical differences in the quality of used substances.
\end{abstract}

Keywords: weak acid salts, warfarin sodium, dissolution method

Dissolution methods for oral drugs containing an active substance in the form of a weak acid salt have to reflect their specific physicochemical and biological properties. In acidic gastric environment, these substance turn into the acid form, which is often poorly soluble. Therefore, dissolution testing in simulated gastric fluid is out of the question. The warfarin sodium tablets USP monograph (warfarin is a typical example of the above mentioned drugs) requires only water as a dissolution medium (1). This method, which is neither biorelevant nor discriminatory because of very good solubility of warfarin sodium in water, contains only one sampling point. This does not allow adequate evaluation of any changes in the quality of the active substance or the impact of different active substance concentrations in tablets of different strengths on the dissolution profile. However, a suitable discriminatory dissolution method can unveil differences in the active substance liberation with respect to its quality and concentration under conditions close to those present in an organism and is thus suitable for estimating in vitro - in vivo correlation (IVIVC) (2).

\footnotetext{
*Correspondence; e-mail: muselikj@vfu.cz
} 
Biphasic dissolution is a suitable dissolution method for drugs in the form of weak acid salts, combining the acidic gastric environment and lipophilic character of cell membranes through which the drug is absorbed. Having partially dissolved in the aqueous acidic phase, the active substance in the form of a weak acid may move by diffusion to the organic layer; finally, a balanced state dependent on the partition coefficient is achieved. Biphasic dissolution system is simple, easy to implement and uses commonly available equipment. The advantage of this system lies in its single-step likeness to in vivo liberation, dissolution and absorption of active substances in the gastrointestinal tract (3). A commercial automated equipment for biphasic dissolution testing has been recently introduced, allowing easy, routine use of this method (4). The aim of this study was to identify suitable conditions for testing substances in the form of weak acid salts, using a model example of tablets containing warfarin sodium in two concentrations and two particle size distributions. The resulting dissolution profile should be similar as much as possible to the in vivo liberation. Furthermore, the proposed method was evaluated to find if it is discriminatory and able to detect changes in both the content and quality of active pharmaceutical ingredients (API).

\section{EXPERIMENTAL}

\section{Materials}

Warfarin standard, 1-octanol, methanol and hydrochloric acid were all obtained from Sigma Aldrich (UK). All materials were of analytical grade and used without further purification. Deionized water was prepared in house. The composition of tested tablets and used raw material is given in Table I.

Table I. Composition of the blends and physical properties of individual constituents

\begin{tabular}{llccccccc}
\hline \multirow{2}{*}{ Component } & Producer & \multicolumn{3}{c}{ Particle size $(\mu \mathrm{m})^{\mathrm{a}}$} & \multicolumn{3}{c}{ Content (\%) } \\
\cline { 3 - 9 } & & $D_{10}$ & $D_{50}$ & $D_{90}$ & W1A & W10A & W1B & W10B \\
\hline $\begin{array}{l}\text { Warfarin } \\
\text { sodium clathrate }\end{array}$ & Pliva (Croatia) & 0.9 & 4.8 & 10.3 & 2.0 & & - \\
Di-cafos 92-14 & 2.7 & 22.5 & 234.3 & - & & 2.0 \\
& $\begin{array}{l}\text { Budenheim KG } \\
\text { (Germany) }\end{array}$ & 2.4 & 152.3 & 309.8 & & 70.0 & \\
Avicel PH 101 & $\begin{array}{l}\text { FMC BioPolymer } \\
\text { (USA) }\end{array}$ & 14.7 & 46.3 & 110.7 & & 25.0 & \\
Ac-Di-Sol & $\begin{array}{l}\text { FMC BioPolymer } \\
\text { (USA) }\end{array}$ & 12.2 & 33.1 & 86.7 & & 2.0 & \\
Magnesium & $\begin{array}{l}\text { Peter Greven } \\
\text { stearate }\end{array}$ & 2.6 & 10.2 & 23.1 & & 2.0 & \\
\hline
\end{tabular}

${ }^{a} D_{x}-x$ is $\%$ of measured particles that are smaller than this size $(\mu \mathrm{m})$.

Di-cafos - calcium hydrogen phosphate, Avicel PH 101 - microcrystalline cellulose, Ac-Di-Sol - sodium crosscarmellose 
A. Franc et al.: Biphasic dissolution method for quality control and assurance of drugs containing active substances in the form of weak acid salts, Acta Pharm. 66 (2016) 139-145.

\section{Manufacturing of tablets}

In order to design the biphasic dissolution method, tablets containing $1 \mathrm{mg}$ or $10 \mathrm{mg}$ of API were manufactured by direct compression from a single tabletting blend. All manufactured batches met the requirements of valid USP and EP monographs on friability, hardness and disintegration. Two batches of each strength were prepared, differing in particle size distribution of API, i.e., batches with 1 or $10 \mathrm{mg}$ of API of narrow particle size distribution (batches W1A and W10A), respectively, and of wide particle size distribution (batches $\mathrm{W} 1 \mathrm{~B}$ and $\mathrm{W} 10 \mathrm{~B})$. The composition of tabletting blends and the manufacturing process are based on the VFU patent (5) and literature (6).

The composition of the blends and some physicochemical properties of raw materials are listed in Table I. All constituents were sieved through a 250- $\mu \mathrm{m}$ sieve. All constituents without magnesium stearate were mixed for 10 minutes, then magnesium stearate was added and another 5 minutes of mixing followed. A Turbula (T2C, Switzerland) homogenizer was used, speed $40 \mathrm{rpm}$. The mass of a batch was $500.0 \mathrm{~g}$.

Tablets containing $1 \mathrm{mg}$ of API weighed $54.2 \mathrm{mg}$. They were flat with a 5 -mm diameter and hardness about $100 \mathrm{~N}$. Tablets containing $10 \mathrm{mg}$ of API weighed $542.0 \mathrm{mg}$; they were flat with a 10-mm diameter and hardness about $100 \mathrm{~N}$. All batches were produced using an eccentric press (Korsch EK0, Germany).

\section{Dissolution test}

The tests were carried out on a SOTAX (AT 7 Donau Lab, Switzerland). Biphasic dissolution was performed using $900 \mathrm{~mL}$ of $0.1 \mathrm{~mol} \mathrm{~L}^{-1} \mathrm{HCl}$ and $70.0 \mathrm{~g}$ of 1-octanol maintained at $37.0 \pm 0.5^{\circ} \mathrm{C}$, using USP paddles at a rotation speed of $50 \mathrm{rpm}$. One $\mathrm{mL}$ of dissolution medium (water and organic phase) was withdrawn after 1,2, 4 and 8 hours of dissolution. Dissolution of tablets in water was done according to the USP monograph using $900 \mathrm{~mL}$ of water maintained at $37^{\circ} \mathrm{C}$ and paddles rotating at $50 \mathrm{rpm}$ (1). Sampling points were at 10, 20,30 and $60 \mathrm{~min}$. Samples from all dissolution tests were filtered using $0.45-\mu \mathrm{m}$ syringe filters; warfarin content was then analyzed by liquid chromatography. For each sampling point, mean and standard deviations were calculated.

\section{Chromatographic analysis}

To measure warfarin content in samples, we used high performance liquid chromatography and adhered to the published method (6). Quantification followed the calibration curve, which expressed the relation between the peak area and warfarin concentration (samples from organic phase) or warfarin clathrate sodium salt concentration (samples from aqueous phase).

\section{Statistical analysis}

To compare the dissolution profiles of tablet batches, the difference factor $f_{1}$ and the similarity factor $f_{2}$ were calculated. Difference and similarity factors were determined for samples W1A-W10A, W1B-W10B to find the influence of warfarin content in tablets (1 mg or $10 \mathrm{mg}$ ) and for samples W1A-W1B, W10A-W10B to examine the effect of warfarin particle size distribution (mean particle size 4.8 or $22.5 \mu \mathrm{m}$ ). 


\section{RESULTS AND DISCUSSION}

In this study, warfarin sodium was chosen as a model drug for the development of a biphasic dissolution method because of its physicochemical properties. Warfarin is used as sodium salt and as free acid; it is acidic $\left(\mathrm{pK}_{\mathrm{a}}=5.0\right)$ and poorly soluble in water. In order to develop a discriminatory dissolution method suitable for evaluation of active substances (weak acids), tablets containing warfarin clathrate sodium salt were prepared (composition in Table I) choosing strengths of commercially available warfarin (1 mg and $10 \mathrm{mg}$ ). Considering the fact that physical properties of API have an impact on warfarin pharmacokinetics (7), different particle size distribution of the active substance was chosen as a qualitative parameter $\left(D_{50}=4.8\right.$ or $\left.22.5 \mu \mathrm{m}\right)$. These two variables were chosen because the optimal dissolution method should identify changes in product quality while being suitable for the whole range of marketed strengths. Manufactured tablets were tested according to EP and USP quality requirements for friability, hardness and disintegration. The results of they were similar, and as these tests were run according to pharmacopoeial monographs, they prove that physical parameters will not have a significant impact on the differences in dissolution profiles. The USP dissolution method was used to evaluate tablets with different warfarin contents and particle size distribution. Fig. 1 shows that after 10 minutes, almost all active substance was liberated in all batches and no differences between samples could be observed. This method is neither discriminatory nor biorelevant.

The conditions of a dissolution method suitable for dosage forms containing active substances in the form of salts of weak acids should simulate real conditions in the gastrointestinal tract as much as possible. Therefore, we chose $0.1 \mathrm{~mol} \mathrm{~L}^{-1}$ hydrochloric acid for the aqueous phase (simulation of gastric environment) and 1-octanol for the organic phase (simulation of transport through the biological barrier). Further dissolution conditions

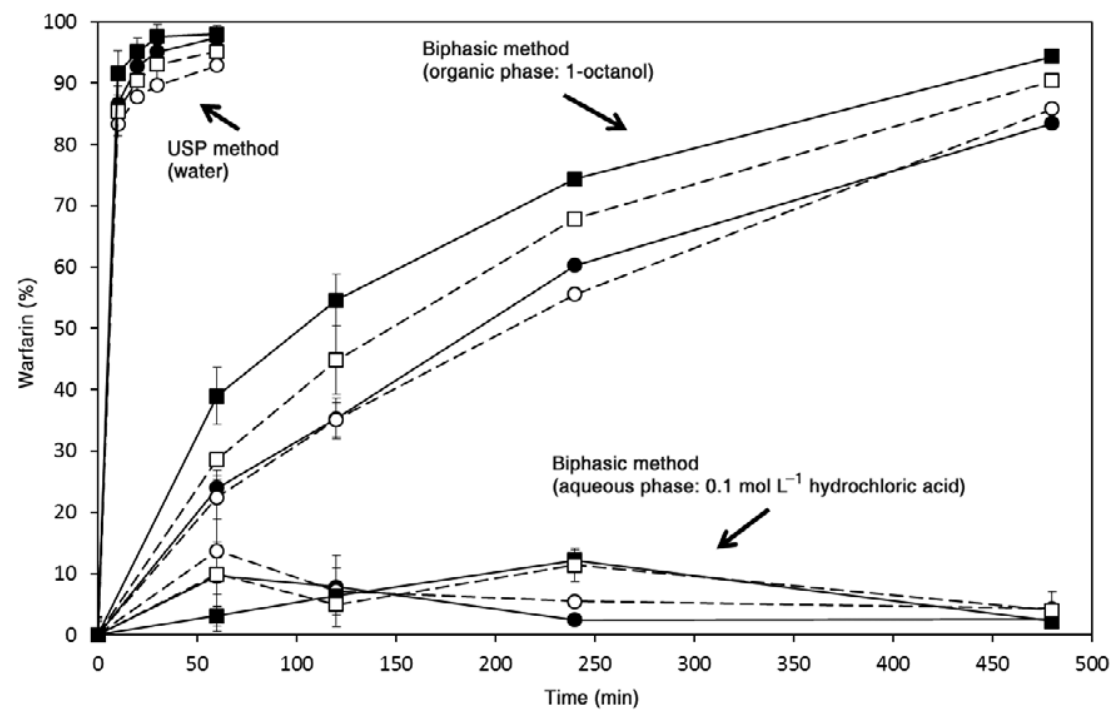

Fig. 1. Dissolution profiles of warfarin tablets under different conditions (USP method and biphasic method):

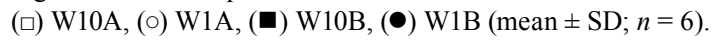


A. Franc et al.: Biphasic dissolution method for quality control and assurance of drugs containing active substances in the form of weak acid salts, Acta Pharm. 66 (2016) 139-145.

such as the volume of aqueous phase, temperature and paddle rotations follow dissolution requirements as given in the Warfarin Sodium Tablets USP monograph (1). As small as possible volume of organic phase was chosen to allow easy sampling and meeting ,sink conditions" (the concentration of saturated solution of warfarin in 1-octanol is five times higher than the concentration of warfarin solution in 1-octanol obtained by dissolving completely the dose contained in one tablet) during the dissolution process of both tested strengths.

At first sight, the dissolution profiles obtained by biphasic and pharmacopoeial methods (Fig. 1) state clearly that dissolution profiles in the 1-octanol phase do differ whereas those acquired by the USP dissolution method do not. Using the similarity factor $f_{2}$ and the difference factor $f_{1}$ for statistical evaluation of the likeness of these profiles (Table II), we found out that the dissolution profiles of tablets with different strengths differ statistically significantly only in the case of API with wide particle size distribution. Dissolution profiles of tablets with different strengths and API with narrow particle size distribution are statistically different when using $f_{1}$ for comparison $\left(f_{1}>15.0\right)$. When using $f_{2}$, there was no statistically significant difference between these profiles; however, $f_{2}$ was close to the critical value of 50 . Under chosen conditions of biphasic dissolution, the method is discriminatory with respect to the content of active substance in the tablet. Statistical comparison of the dissolution profiles of tablets with the same strength and different particle size distribution of API has proven that these dissolution profiles are similar (according to both $f_{2}$ and $f_{1}$ ). Nevertheless, the actual values of $f_{2}$ and $f_{1}$ show that the impact of particle size distribution on the dissolution profile is higher in tablets with higher API content $\left(f_{2}=54.5\right.$, $\left.f_{1}=11.7\right)$ than in tablets with lower content of API $\left(f_{2}=76.7, f_{1}=4.3\right)$. Different contents of API in tablets led to more pronounced differences in dissolution profiles than physical properties, i.e., particle size distribution of the active substance.

In the course of biphasic dissolution testing, the concentration of API in the aqueous phase $\left(0.1 \mathrm{~mol} \mathrm{~L}^{-1} \mathrm{HCl}\right)$ was measured as well. In all batches, the content of API in the aqueous phase was lower than $15 \%$ (Fig. 1). During the dissolution test, the non-ionized active substance is moving continually to the lipophilic phase, similarly to the process of absorption in the gastrointestinal tract. This finding is in accord with the requirements of a biorelevant dissolution method.

The described biphasic dissolution method meets the requirements for the calculation and use of $f_{2}$ and $f_{1}$ factors with respect to the number of sampling points (60, 120, 240 and 480 minutes), RSD values (lower than $20 \%$ at the first point, lower than $10 \%$ at subsequent points, Table III), and only one point lies outside the line of $85 \%$ of dissolved active substance. Owing to the character of the dissolution method, which takes into account physi-

Table II. Biphasic dissolution method: difference factor and similarity factor

\begin{tabular}{lccc}
\hline Sample & Reference & $f_{2}$ & $f_{1}$ \\
\hline W10A & W1A & 52.8 & 16.5 \\
W10B & W1B & 40.9 & 29.3 \\
W10A & W10B & 54.5 & 11.7 \\
W1A & W1B & 76.7 & 4.3 \\
\hline
\end{tabular}


A. Franc et al:: Biphasic dissolution method for quality control and assurance of drugs containing active substances in the form of weak acid salts, Acta Pharm. 66 (2016) 139-145.

ological conditions, this method meets general requirements for the design of dissolution methods. This method can be considered biorelevant, since $50 \%$ of active substance is released in 120-240 minutes, corresponding to the peak concentration of warfarin in blood plasma in vivo, i.e., 108-222 min after oral administration (7). The method seems to be suitable for IVIVC (8).

Table III. Variability of biphasic dissolution profiles in 1-octanol

\begin{tabular}{lcccc}
\hline \multirow{2}{*}{ Sample } & \multicolumn{4}{c}{ RSD obtained from dissolution data time intervals (min) (\%) } \\
\cline { 2 - 5 } & 60 & 120 & 240 & 480 \\
\hline W10A & 17.6 & 5.8 & 1.5 & 6.4 \\
W10B & 18.0 & 4.3 & 6.6 & 4.7 \\
W1A & 11.4 & 8.2 & 6.6 & 3.4 \\
W1B & 14.1 & 8.4 & 2.4 & 4.1 \\
\hline
\end{tabular}

\section{CONCLUSIONS}

A biphasic discriminatory dissolution method was designed based on using the upper organic layer (1-octanol) and the lower aqueous layer $\left(0.1 \mathrm{~mol} \mathrm{~L}^{-1} \mathrm{HCl}\right)$, which simulates the gastric environment. The method seems to be suitable for dosage forms containing active substances in the form of salts of weak acids, where dissolution testing in simulated gastric fluid is inconvenient. The designed conditions of biphasic dissolution testing are sufficient to meet the requirements for $f_{1}$ and $f_{2}$ calculation. The method is suitable for statistical comparison of the dissolution profiles of tablets with different quantitative or qualitative parameters and method for the development, clinical evaluation, as well as routine quality control release evaluation. As the equipment for routine biphasic dissolution measurement is already available, this method could play a major role in quality assurance and control as well as help decrease development costs for both innovative and generic pharmaceutical industry.

Acknowledgements. - This work was supported by project IGA VFU Brno 55/2014/FaF.

\section{REFERENCES}

1. United States Pharmacopoeia 38 - National Formulary 33, USP Convention, Rockville (MD) 2015, p. 3869.

2. S. Klein, The use of biorelevant dissolution media to forecast the in vivo performance of a drug, AAPS. J. 12 (2010) 397-406; DOI: 10.1208/s12248-010-9203-3.

3. Y. Qiu, Y. Chen, G. Zhang and W. Porter, Developing Solid Oral Dosage Forms: Pharmaceutical Theory and Practice, Academic Press, London 2009, p. 411.

4. Sirius Analytical Instruments Ltd., New instrument for automated biorelevant biphasic dissolution and solubility testing, Eur. Pharmaceut. Rev. October 2013; http://www.americanpharmaceu- 
ticalreview.com/22313/news/featured-news-instrument-automated-biorelevant-biphasic-dissolution-solubility-testing/; last access date September 24, 2015.

5. A. Franc and J. Muselik, Method for Preparing Solid Drug from Warfarin Sodium Salt in Form of Isopropanol Clathrate, Involves Determining Total Weight of Solid Drug and Distribution Ratio of Warfarin Sodium Salt, Pat. CZ304136-B6, October 2013.

6. J. Muselik, A. Franc, P. Dolezel, R. Gonec, A. Krondlova and I. Lukasova, Influence of process parameters on content uniformity of a low dose active pharmaceutical ingredient in a tablet formulation according to GMP, Acta Pharm. 64 (2014) 355-367; DOI: 10.2478/ACPH-2014-0022.

7. L. M. Vercaigne and G. G. Zhanel, Clinical significance of bioequivalence and interchangeability of narrow therapeutic range drugs: focus on warfarins, J. Pharm. Pharm. Sci. 1 (1998) 92-94.

8. V. R. S. Uppoor, Regulatory perspectives on in vitro (dissolution)/in vivo (bioavailability) correlations, J. Control. Release 72 (2001) 127-132; DOI: 10.1016/S0168-3659(01)00268-1. 